\title{
A Novel Circuit Topology for Underwater Wireless Power Transfer
}

\author{
Francisco Gonçalves, Cândido Duarte, and L. M. Pessoa \\ INESC TEC and Faculty of Engineering, University of Porto \\ Porto, Portugal \\ Email: francisco.goncalves@fe.up.pt, candidoduarte@fe.up.pt, lpessoa@inesctec.pt
}

\begin{abstract}
This work presents a new topology for the matching networks of an underwater wireless power transfer system. A class-D driver is used in resonance at fundamental and third harmonic frequencies. The double resonance helps reducing the reverse voltage stress at the diode rectifier. We present the analytical derivation of the proposed network and demonstrate the design procedure with an example. We also show that additional degrees of freedom can be acquired with the proposed topology, which improves the design space for time-varying operation conditions of our application, such as the load changing when a battery is being recharged. The performance of our topology is compared to most conventional approaches, such as the series-series and series-parallel networks, revealing a good compromise between power delivery and efficiency across a wide load range.
\end{abstract}

Keywords-coupling circuits; inductive power transmission; loosely coupled coils; resonant inverters; wireless power;

\section{INTRODUCTION}

Despite the concept of wireless power transfer (WPT) being around since the beginning of the 20th century (with the pioneer work of Nikola Tesla [1], [2]), only during the past decade the idea had met substantial developments [3][7]. WPT has been revived with a new diversity of applications, such as battery refilling systems for electric vehicles [6], powering of RFID tags [8], and battery charging of consumer electronics or implantable medical devices [3], [9]. In our case, the purpose of application is to recharge a small-scale autonomous underwater vehicle (AUV) in saline waters [10] (Fig. 1). The use of underwater WPT (UWPT) alleviates the need of human assistance for plugging in and out electrical connectors in the docking station. Instead, the AUV batteries can be recharged at deep underwater with reduced maintenance demands, thereby improving the autonomy of the vehicle.

Recent WPT systems rely on non radiative power using magnetically coupled coils separated at some distance (from $\mathrm{mm}$ to several $\mathrm{cm}$ ), operating at resonance [4], [5]. In these systems, the most common approaches for driver architectures are: the half-bridge inverter (class D), class-E inverter, and current-mode driver (inverse class D). Although the current-mode driver yields zero-voltage switching (ZVS), it has the disadvantage of an excessive voltage stress at the drains of the transistors, hence degrading the power utilization factor [11]. As for the class-E inverter, besides

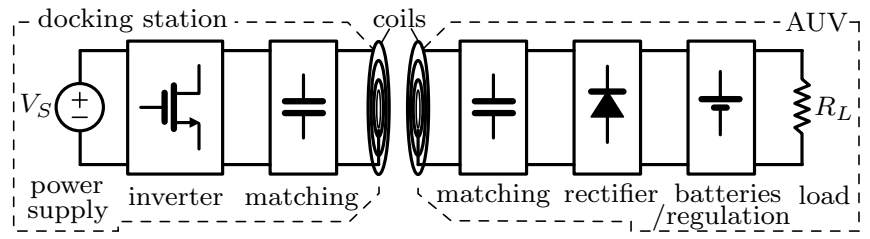

Figure 1. UWPT system configuration for charging the batteries of an AUV.

ZVS, in its typical operation it also allows zero-voltagederivative switching (ZVDS) [12], and produces more than one and a half output power than the class D for the same voltage supply and load [7]. However, again this comes at the expense of an increased peak voltage at the drain, which is more than three times its supply voltage. Another obstacle in the use of the class-E driver is the high sensitivity to load resistance variations [13] that brings the operation to regimes where ZVS and ZVDS conditions cannot be achieved.

In power applications, the class-D inverter is the most classical approach, essentially due to its simplicity in design and control [14], and for having no implications on the voltage stress (since the switching-node peak voltage equals the supply-voltage value). Typically, in a resonant inductive driver, the class-D inverter makes use of one of two different matching networks connected to the coils, namely the seriesseries and series-parallel configurations [7]. The former topology consists on a capacitor added in series to each coil, whereas the latter differs only on the receiver side, with the second capacitor connected in parallel with the load [15]. Both approaches might not provide reasonable degrees of freedom in terms of design space to mitigate the compromise between power delivery and efficiency. Moreover, it is not simple to accommodate a wide range of certain parameters. Since any WPT system will vary either at the distance between coils (with direct impact on the coupling factor) or the load (or even both), design strategies are required to overcome an expected variability. The lack of topological alternatives is also critical particularly when the design of the coils for the inductive link is carried out apart from the remaining system, i.e. the driver and rectifier optimization procedures are constrained by the coils chosen. In the present work we introduce a new coupling network for existent coils and optimize the system for a square wave voltage excitation 


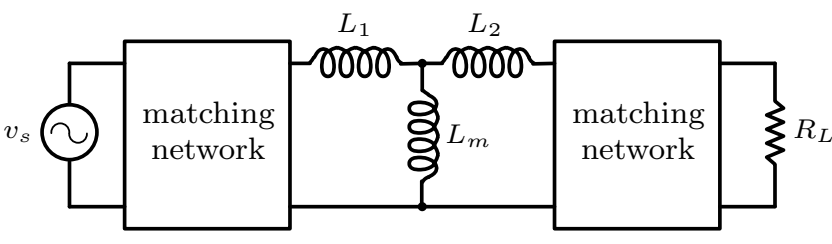

Figure 2. UWPT system with the "tee" model of coupling coils.

from a class-D driver. The proposed network topology is suitable for UWPT, being able to optimize the load variation due to the charge phase of the batteries at the rectifier side. The paper is organized as follows. The following section addresses the UWPT system, the proposed topology is presented next, in section III, and then simulation results for the proposed approach are presented in section IV.

\section{UWPT SYSTEM DESCRIPTION}

When the AUV arrives at the docking station, a retention mechanism is set to keep the vehicle still at a fixed position, minimizing possible fluctuations and settling the adequate alignment between the AUV and the recharging system. This means we can assure a nearly constant (although loose) coupling factor $(k)$ during the recharge phase $(k \simeq 0.304$ for a distance of $4 \mathrm{~cm}$ between coils). On the other hand, the highly conductive properties of the seawater as transfer medium prevent the usage of high frequencies for UWPT operation. The operating frequency was chosen at $f_{0}=$ $100 \mathrm{kHz}$ and spiral coupling coils were manufactured in order to achieve their maximum unloaded quality factor around $f_{0}$. The coils are represented by the equivalent circuit of a transformer comprised by $L_{1}, L_{2}$ and $L_{m}$, as depicted in Fig. 2.

The inductors $L_{1}$ and $L_{2}$ are equal-valued self inductances, and $L_{m}$ represents the mutual inductance. The coil parameters for the UWPT system are given below

$$
\begin{aligned}
L & =18.25 \mu \mathrm{H} \\
L_{1}=L_{2} & =(1-k) \cdot L=12.7 \mu \mathrm{H} \\
L_{m} & =k \cdot L=5.548 \mu \mathrm{H}
\end{aligned}
$$

In Fig. 2, $R_{L}$ represents the input impedance of the rectifier. In fact, this value should reflect the current required by the charging process of the batteries (hence, $R_{L}$ varies in time) and should be influenced by the conduction angle of the rectification diode as well [16]. As such, both matching networks must be carefully designed according to the load profile.

At the other end, the source $v_{s}$ represents the input excitation, given here as a sinusoidal voltage. Some works optimize the coupling network assuming such a sinusoidal input and for demonstration purposes validate it using a vector network analyzer, but for a more realistic scenario the driver will apply either square voltage or current waveforms at the input (or other formats, but rarely sinusoidal). For the

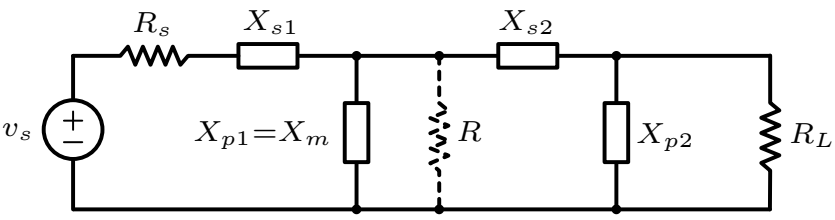

Figure 3. Cascade L network as the basis to develop the proposed topology.

reasons referred earlier in introduction, we will make use of a class-D inverter, hence in such a case $v_{s}$ in Fig. 2 then represents a square wave at $f_{0}$. The design of the remaining UWPT system will be constrained to the specifications of the coupling coils mentioned above.

\section{Proposed Circuit Topology}

Since the square wave at the output of the inverter comprises non-null odd harmonics, for the proposed circuit instead of establishing resonance only at the fundamental frequency $f=f_{0}$, we will provide resonance at $f=3 f_{0}$ as well. This brings the benefit of lowering the reverse peak voltage at the rectifier diode and, as a consequence, it allows us to increase the power supply at the inverter if there is still some margin below the maximum voltage ratings of the transistors. We will make use of low loaded qualityfactor $(Q)$ networks [17], aiming at broadband matching to easier the finding a solution for simultaneous resonance. Fig. 3 depicts a cascade L network, in which we make the parallel reactance of the first $\mathrm{L}$ leg coincident with the mutual inductance of our coupling coils. The resistor $R$ represents a "virtual resistor", which will be used as basic design parameter. Its value can be arbitrated in a range limited by $R_{s}$ and $R_{L}$. If we consider $R_{s}<R<R_{L}{ }^{1}$, then $Q$ is given by

$$
Q=\frac{R}{\omega_{0} L_{m}}=\sqrt{\frac{R_{L}}{R}-1}
$$

where $\omega_{0}=2 \pi f_{0}$. The equation above leads to

$$
R^{3}-\left(\omega_{0} L_{m}\right)^{2}\left(R_{L}-R\right)=0
$$

The solution for (5) is plotted in Fig. 4 admitting (3). For a given $R_{L}$ profile one can choose $R$ from (5) to derive the remaining components.

Let us go back to Fig. 3 where we assume that the reactance $X_{p 2}$ denotes a capacitor in parallel with the load, i.e. $X_{p 2}=1 /\left(\omega_{0} C_{p 2}\right)$ and

$$
C_{p 2}=\frac{Q}{\omega_{0} R_{L}}
$$

\footnotetext{
${ }^{1}$ The other possibility $R_{L}<R<R_{s}$ is not addressed here because it leads to a solution in which $R_{L}<1 \Omega$, i.e. in the order of the equivalent series resistance of inductors, which can be reflected in a low power efficiency.
} 


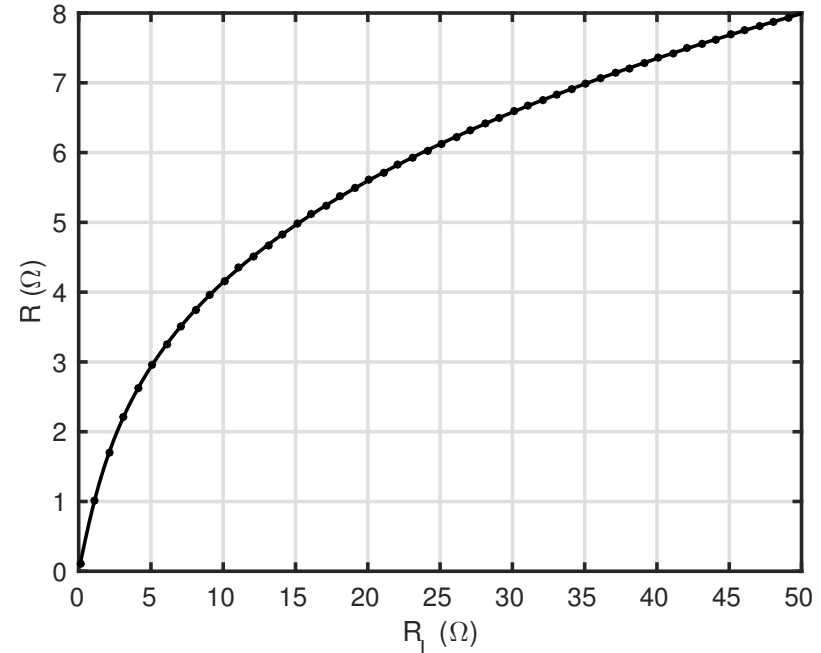

Figure 4. Virtual resistance $R$ vs load resistance $R_{L}$ for $\omega_{0} L_{m} \simeq 3.486 \Omega$.

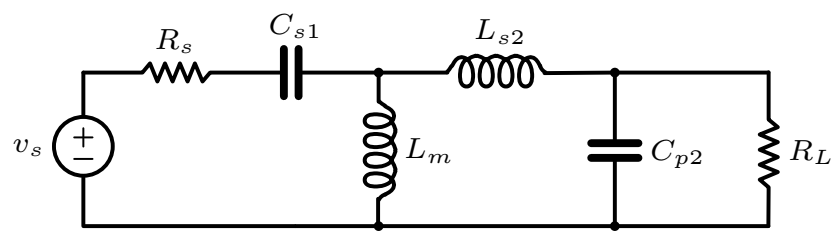

(a)

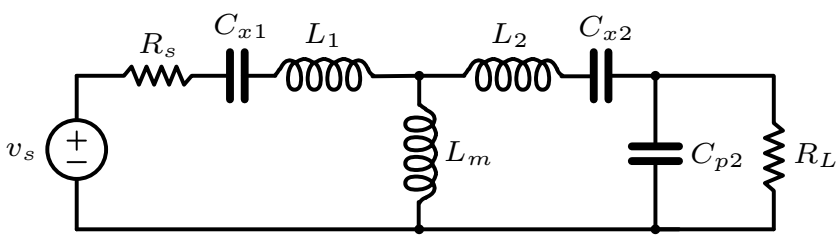

(b)

Figure 5. Basic network to develop the proposed topology: (a) cascade $\mathrm{L}$ network; and (b) modified network to include the coupling coils.

One should use an inductor to complete the second L leg at $X_{s 2}$. The respective reactance is determined as follows

$$
X_{s 2}=R \cdot Q
$$

Fig. 5(a) depicts the arbitrated passives in place of the reactances shown in Fig. 3. However, since in fact we have a transformer in the coupling network, we need to compensate for the existent inductance, $L_{2}$. Therefore, admitting $X_{s 2}<\omega_{0} L_{2}$, we need to add in series $C_{x 2}$

$$
C_{x 2}=\frac{1}{\omega_{0}^{2} L_{2}-\omega_{0} R Q}
$$

As for $X_{s 1}$, first an equivalent series resistance is determined to maximize the bandwidth, i.e. imposing $R$ as the geometric mean of $R_{s}$ and $R_{L}$ [17], from which results

$$
R_{s}=\frac{R^{2}}{R_{L}}
$$

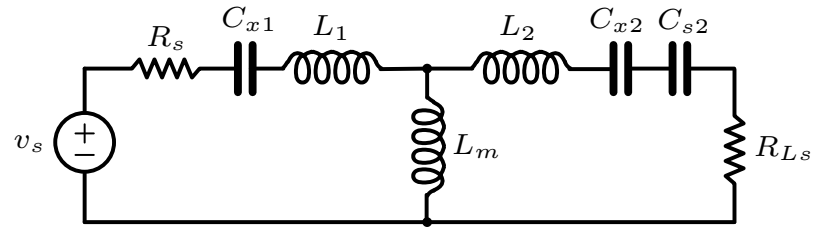

(a)

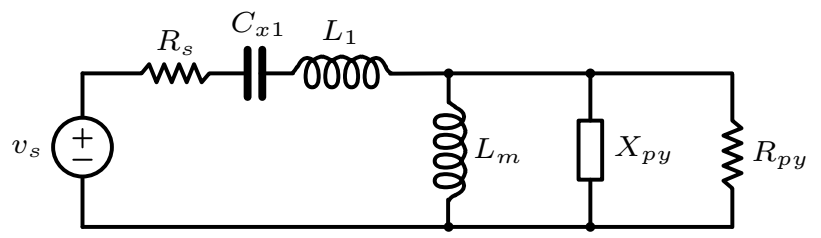

(b)

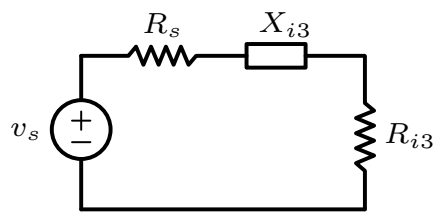

(c)

Figure 6. Simplifications for the circuit in Fig. 5(b) valid only for $f=3 f_{0}$.

then

$$
X_{s 1}=\frac{R_{L}}{R^{2}} Q
$$

Since there is already $L_{1}$ from the coupling coil, one should compensate it to achieve the required $X_{s 1}$. This means a capacitor is needed in series with the value given next

$$
C_{x 1}=\frac{1}{\omega_{0}^{2} L_{1}+\omega_{0} \frac{R^{2}}{R_{L}} Q}
$$

Fig. 5(b) shows the complete network for which, according to the procedure just given, the impedance seen from $v_{s}$ should be $2 R_{s}$ at $f=f_{0}$.

In order to achieve resonance also at $f=3 f_{0}$, we proceed with successive impedance transformations in order to achieve a simplified circuit seen from the input. We start by noting that

$$
Q_{3}=3 \omega_{0} R_{L} C_{p 2}=3 Q
$$

and proceed with the first parallel-to-series transformations and vice versa. Table I summarizes all the intermediate steps and its correspondence to Fig. 6. We make use of the required equality for the quality factor of parallel and series networks, i.e. $Q_{p}=Q_{s}$, let us denote here by $\bar{Q}$. Hence, in terms of resistance: $R_{p}=R_{s}\left(1+\bar{Q}^{2}\right)$; and for the reactance: $X_{p}=X_{s}\left(1+1 / \bar{Q}^{2}\right)$. The subscripts "s" and "p" denote either "series" or parallel, respectively.

Fig. 6(a) shows the first step of the simplification. The subsequent procedure is a series-to-parallel transformation that leads to Fig. 6(b). Fig. 6(c) corresponds to the most simple circuit, with a load input equivalent resistance $R_{i 3}$ at $f=3 f_{0}$, and similarly an equivalent reactance $X_{i 3}$. 


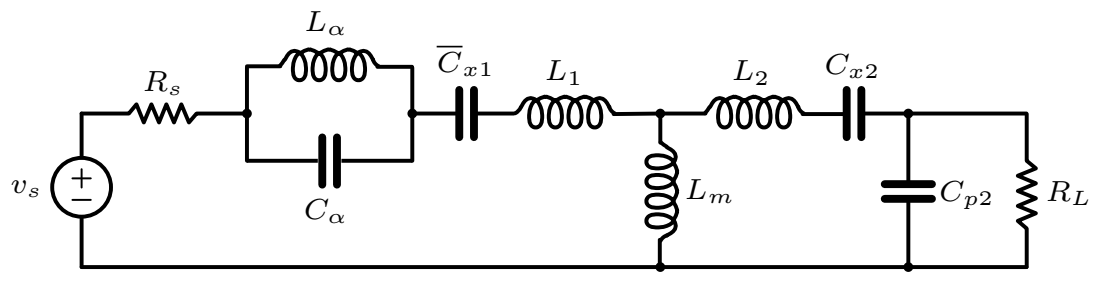

Figure 7. Circuit model for the proposed network topology.

TABLE I

SIMPLIFICATION EXPRESSIONS FOR THE CIRCUIT AT $f=3 f_{0}$.

\begin{tabular}{lll}
\hline & \multicolumn{1}{c}{$C_{s 2}$} & $\left(1+1 / Q_{3}^{2}\right) C_{p 2}$ \\
\cline { 2 - 2 } Fig. 6(a) & $R_{L s}$ & $\frac{1}{1+Q_{3}^{2}} R_{L}$ \\
\cline { 2 - 2 } & $C_{x s 2}$ & $\frac{C_{x 2} C_{s 2}}{C_{x 2}+C_{s 2}}$ \\
\hline & $X_{s y}$ & $3 \omega_{0} L_{2}-\frac{1}{3 \omega_{0} C_{x s 2}}$ \\
\cline { 2 - 3 } Fig. 6(b) & $Q_{y}$ & $X_{y s} / R_{L s}$ \\
\cline { 2 - 2 } & $R_{p y}$ & $R_{L s}\left(1+Q_{y}^{2}\right)$ \\
\cline { 2 - 2 } & $X_{p y}$ & $X_{s y}\left(1+1 / Q_{y}^{2}\right)$ \\
\hline & $X_{j}$ & $\frac{3 \omega_{0} L_{m} X_{p y}}{3 \omega_{0} L_{m}+X_{p y}}$ \\
\hline & $Q_{j}$ & $R_{p y} / X_{j}$ \\
\cline { 2 - 2 } & $R_{i 3}$ & $\frac{R_{p y}}{1+Q_{j}^{2}}$ \\
\cline { 2 - 2 } & $X_{i 3}$ & $X_{j} \frac{Q_{j}^{2}}{1+Q_{j}^{2}}+3 \omega_{0} L_{1}-\frac{1}{3 \omega_{0} C_{x 1}}$ \\
\hline
\end{tabular}

Hence, for the desired resonance, $X_{i 3}=0$. To achieve this condition, we add in series with the input a parallel LC tank $\left(L_{\alpha} C_{\alpha}\right.$ ), as shown in Fig. 7. Let us consider $1<\alpha<3$ so that

$$
\left(\alpha \omega_{0}\right)^{2}=\frac{1}{L_{\alpha} C_{\alpha}}
$$

The key idea here is to force an open circuit at a frequency $f=\alpha f_{0}$, between the fundamental and the third harmonic. The impedance added by the LC tank is

$$
Z_{\alpha}(j \omega)=\frac{j \omega L_{\alpha}}{1-\left(\frac{\omega}{\alpha \omega_{0}}\right)^{2}}
$$

which means that $Z_{\alpha}(j \omega)$ is inductive at $\omega=\omega_{0}$ and capacitive at $\omega=3 \omega_{0}$. At the fundamental frequency we need to add a negative reactance in series $Z_{a}$ to establish resonance at $\omega=\omega_{0}$, i.e.

$$
Z_{a}(j \omega)=\frac{1}{j \omega C_{a}}
$$

and

$$
\begin{aligned}
& \frac{1}{j \omega_{0} C_{a}}+j \frac{\omega_{0} L_{\alpha}}{1-1 / \alpha^{2}}=0 \\
& C_{a}=\left(1-1 / \alpha^{2}\right) \cdot \frac{1}{\omega_{0}^{2} L_{\alpha}}
\end{aligned}
$$

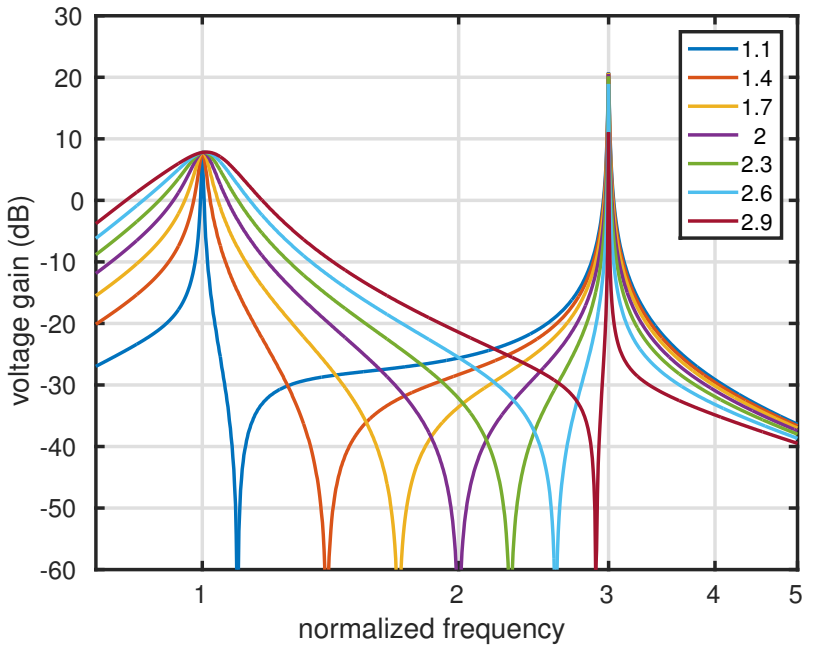

Figure 9. Output voltage for different values of $\alpha$ when the network is excited by an AC voltage source.

This can be included in $C_{x 1}$. Let us denote it as $\bar{C}_{x 1}$,

$$
\bar{C}_{x 1}=\frac{C_{x 1} \cdot C_{a}}{C_{x 1}+C_{a}}
$$

So, in order to have $X_{i 3}=0$,

$$
X_{i 3}+Z_{\alpha}\left(j 3 \omega_{0}\right)-\frac{1}{3 \omega_{0} C_{a}}=0
$$

which, replacing $C_{a}$ by its dependence on $L_{\alpha}$ and developing it further, leads to

$$
L_{\alpha}=\frac{3}{8} \cdot \frac{X_{i 3}}{\omega_{0}} \cdot \frac{\left(1-\alpha^{2}\right)\left(\alpha^{2}-9\right)}{\alpha^{4}}
$$

Fig. 9 depicts the response (voltage at $R_{L}$ ) to a $1 \mathrm{~V}$ signal excitation at the input of the circuit $\left(v_{s}\right.$ in Fig. 7) when the frequency is swept, for different values of $\alpha$ (this is in fact equivalent to $S_{21}$ ). There is a clear trade-off between the bandwidth at $f_{0}$ and $3 f_{0}$. To ensure reasonable insensitivity to frequency deviations $\alpha$ can be chosen with values 1.7 to 2.3. It should be mentioned also that although at $3 f_{0}$ the voltage gain is superior to the case $f=f_{0}$, in a square waveform the third harmonic is much smaller than the fundamental component $(\simeq-9.5 \mathrm{~dB})$. 


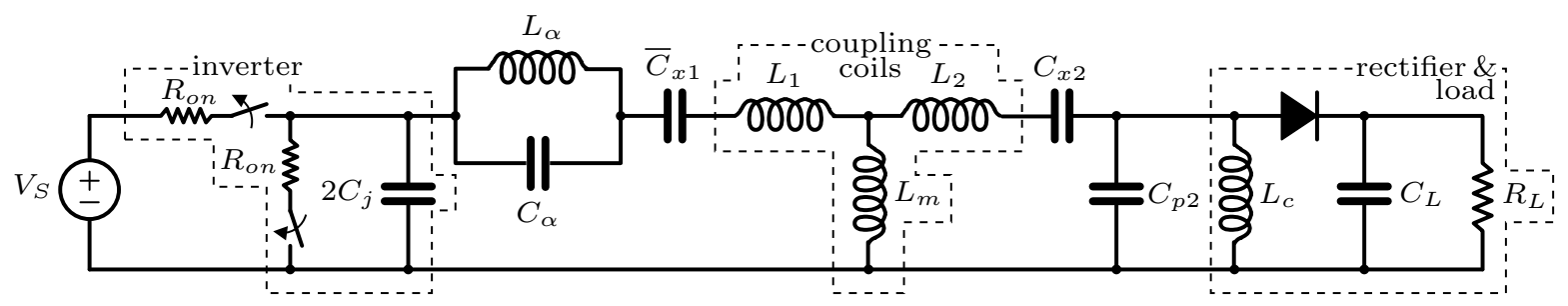

Figure 8. Complete UWPT topology.

TABLE II

PARAMETER VALUES ACCORDING TO THE VALUES ARBITRATED IN (1)-(3), $f_{0}=100 \mathrm{kHz}, R_{L}=10 \Omega, \alpha=2$.

\begin{tabular}{ccc}
\hline Parameter & Value & Unit \\
\hline$R$ & 4.144 & $\Omega$ \\
$R_{s}$ & 1.717 & $\Omega$ \\
$Q$ & 1.189 & - \\
$\boldsymbol{C}_{\boldsymbol{p} \mathbf{2}}$ & $\mathbf{1 8 9 . 2 0}$ & $\mathbf{n F}$ \\
$L_{s 2}$ & 7.840 & $\mu \mathrm{H}$ \\
$\boldsymbol{C}_{\boldsymbol{x} \mathbf{2}}$ & $\mathbf{5 2 1 . 0 1}$ & $\mathbf{n F}$ \\
$C_{x 1}$ & 158.80 & $\mathrm{nF}$ \\
\hline$Q_{3}$ & 3.566 & - \\
$C_{s 2}$ & 204.07 & $\mathrm{nF}$ \\
$R_{L s}$ & 0.729 & $\Omega$ \\
$C_{x s 2}$ & 146.64 & $\mathrm{nF}$ \\
$X_{s y}$ & 20.325 & $\Omega$ \\
$Q_{y}$ & 27.883 & $\bar{\Omega}$ \\
$R_{p y}$ & 567.44 & $\Omega$ \\
$X_{p y}$ & 20.351 & $\Omega$ \\
$X_{j}$ & 6.908 & $\Omega$ \\
$Q_{j}$ & 82.143 & - \\
$R_{i 3}$ & 0.084 & $\Omega$ \\
$L_{i 3}$ & 14.594 & $\mu \mathrm{H}$ \\
\hline $\boldsymbol{L}_{\boldsymbol{\alpha}}$ & $\mathbf{1 5 . 3 9 2}$ & $\boldsymbol{\mu H}$ \\
$\boldsymbol{C}_{\boldsymbol{\alpha}}$ & $\mathbf{4 1 . 1 4 2}$ & $\mathbf{n F}$ \\
$C_{a}$ & 123.43 & $\mathrm{nF}$ \\
$\overline{\boldsymbol{C}}_{\boldsymbol{x} \mathbf{1}}$ & $\mathbf{6 9 . 4 4 8}$ & $\mathbf{n F}$ \\
\hline
\end{tabular}

\section{RESUlts}

Fig. 8 depicts the complete UWPT system, including the class-D inverter and the rectifier. For simulation purposes, the transistors in the inverter are modelled as switches operating in opposite phases, each one having a finite conduction resistance $R_{o n}$ and output capacitance $C_{j}$. At the load, a Schottky barrier rectifier is employed, with $L_{c}$ as a choke to provide a dc path, and a large $C_{L}$ to filter the ripple at $R_{L}$. Table II shows the parameter values for a network designed according to the procedure presented in last section. Spice models for the components (diode MBRS540T3 from Onsemi) were used in the Cadence Virtuoso analog design environment to simulate the proposed circuit topology using Cadence Spectre simulator. Series-series and series-parallel topologies were also included in simulations for reference.

Figs. 10(a) and (b) show the results for the efficiency and power delivery for a load sweep (each line correspond to a different set of component values). The thick black line corresponds to the design of the topology with $R_{L}=10 \Omega$ $(R=4.144 \Omega)$, as shown in Table II. Based on this first

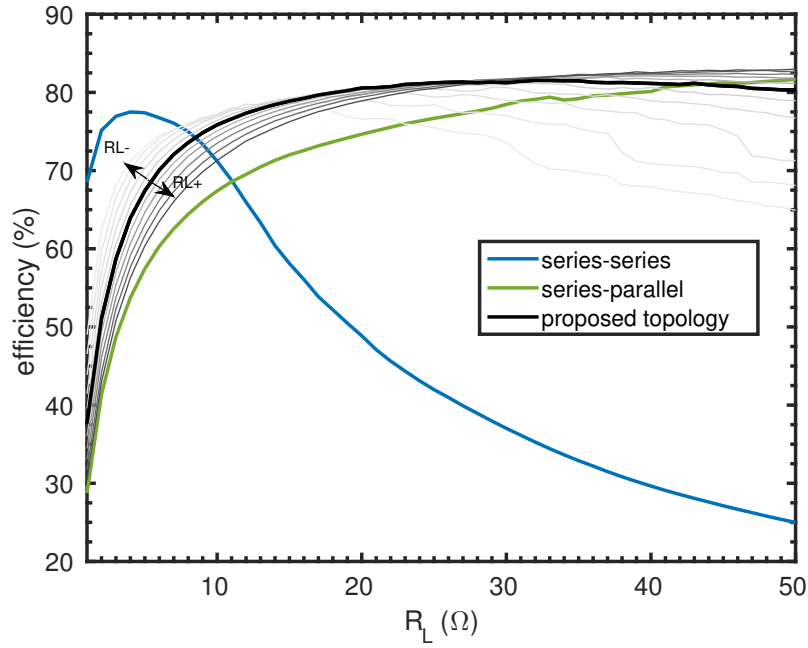

(a)

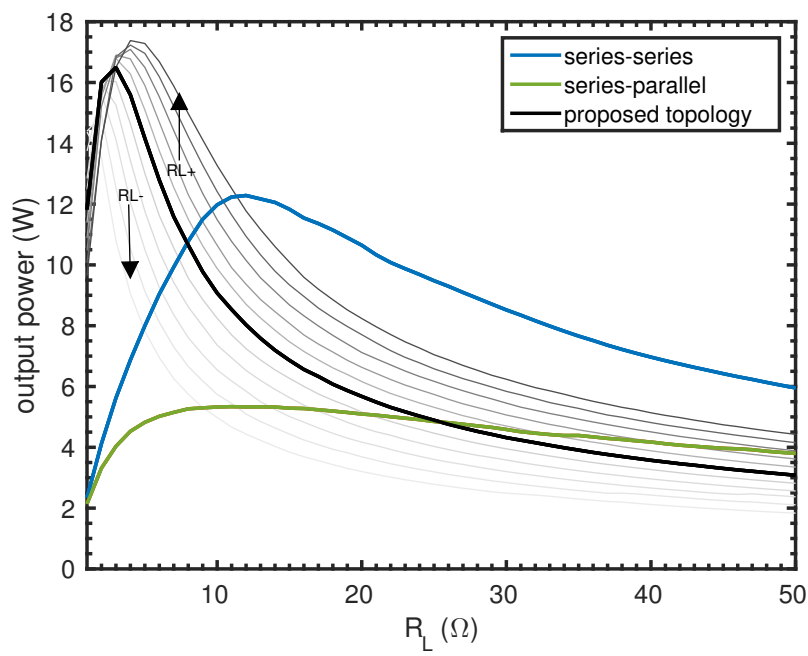

(b)

Figure 10. Simulation results for (a) power efficiency, and (b) power delivery to the load. $R_{L}^{-}$and $R_{L}^{+}$indicate that, in (5), $R_{L} \in[5: 9]$ and $R_{L} \in$ [11:15], respectively.

choice, the compromise between power-delivery and efficiency may be changed - the thinner lines around correspond to deviations up and down to $\pm 50 \%$, denoted by $R_{L}^{+}$and $R_{L}^{-}$. All the simulations were performed with fixed power supply $(10 \mathrm{~V})$ and fixed duty ratio $(50 \%)$ for the switches. 


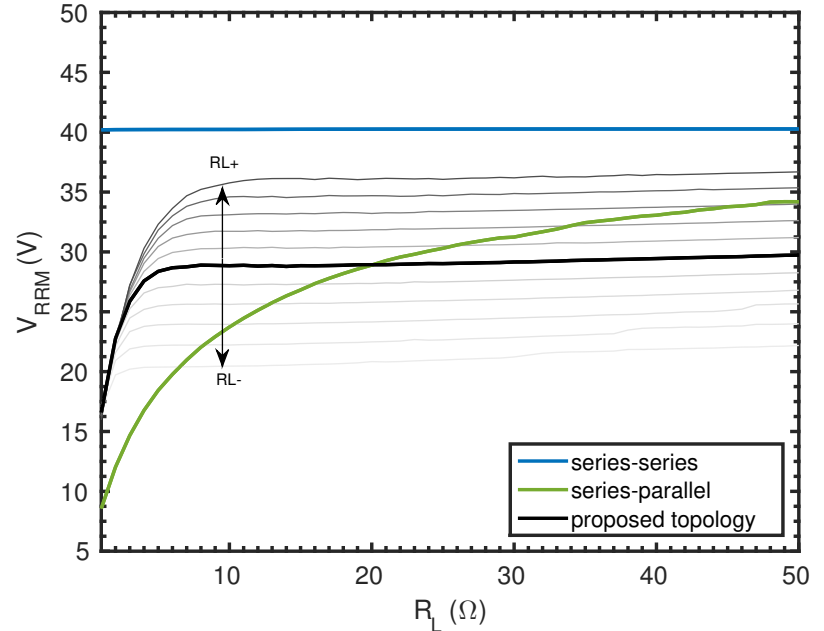

Figure 11. Maximum repetitive reverse voltage $\left(V_{R R M}\right) . R_{L}^{-}$and $R_{L}^{+}$ keep the same meaning as in Fig. 10.

TABLE III

PERFORMANCE COMPARISON FOR $R_{L}=10 \Omega$ AND EQUAL $V_{R R M}$.

\begin{tabular}{r|c|c|c|c}
\hline topology & power supply & efficiency & output power & $V_{R R M}$ \\
\hline series-series & $10 \mathrm{~V}$ & $71.21 \%$ & $11.98 \mathrm{~W}$ & $40.23 \mathrm{~V}$ \\
series-parallel & $17 \mathrm{~V}$ & $68.10 \%$ & $15.51 \mathrm{~W}$ & $40.22 \mathrm{~V}$ \\
proposed & $14 \mathrm{~V}$ & $75.65 \%$ & $17.58 \mathrm{~W}$ & $40.24 \mathrm{~V}$ \\
\hline
\end{tabular}

To better infer about the voltage at the diode, the maximum repetitive reverse voltage $\left(V_{R R M}\right)$ was obtained for each operation condition. Fig. 11 depicts the simulation results, denoting less $V_{R R M}$ required in the proposed topology. Thus, if there is still room available at the driver side, the power supply can be increased, improving also the power delivery. We explored such a case with $R_{L}=10 \Omega$. In the proposed topology, we increased the power supply until we achieved the same $V_{R R M}$ at the diode as in the series-series topology shown in Fig. 11. The results are summarized in Table III, indicating more power at the load for the same voltage stress at the diode rectifier when using our topology.

\section{CONCLUSion}

This paper presents a new approach for designing the matching networks for the driver and rectifier of an UWPT system. Besides the usual resonance at the driver switching frequency, we also establish a resonance at the third harmonic, which mitigates the diode reverse voltage stress at the rectifier. This is possible with the use of an adequate network synthesis with modified cascade $\mathrm{L}$ networks to prevent too sharp bandwidths around resonances. We described a methodology for parametric derivation that tackles the usual compromise between efficiency and power delivery, when compared to conventional approaches, such as series-series and series-parallel topologies. This is particularly important in an underwater application where the saline water medium restricts the coil design.

\section{ACKNOWLEDGMENT}

This work has been developed as part of project ENDURE - Enabling Long-Term Deployments of Underwater Robotic Platforms in Remote Oceanic Locations (PT02 Aviso4 0015) benefiting from a $218157 €$ grant from Iceland, Liechtenstein and Norway through the EEA Grants.

\section{REFERENCES}

[1] N. Tesla, "Method of intensifying and utilizing effects transmitted through natural media." Nov 1901, US Patent 685,953.

[2] — "Apparatus for utilizing effects transmitted from a distance to a receiving device through natural media." Nov 1901, US Patent 685,955 .

[3] B. Lee, M. Kiani, and M. Ghovanloo, "A triple-loop inductive power transmission system for biomedical applications," IEEE Trans. Biomed. Circuits Syst., vol. 10, no. 1, pp. 138-148, Feb 2016.

[4] J. Dai and D. C. Ludois, "A survey of wireless power transfer and a critical comparison of inductive and capacitive coupling for small gap applications," IEEE Trans. Power Electron., vol. 30, no. 11, pp. 6017-6029, Nov 2015.

[5] S. Y. R. Hui, W. Zhong, and C. K. Lee, "A critical review of recent progress in mid-range wireless power transfer," IEEE Trans. Power Electron., vol. 29, no. 9, pp. 4500-4511, Sep 2014.

[6] J. Shin, S. Shin, Y. Kim, S. Ahn, S. Lee, G. Jung, S. J. Jeon, and D. H. Cho, "Design and implementation of shaped magneticresonance-based wireless power transfer system for roadway-powered moving electric vehicles," IEEE Trans. Ind. Electron., vol. 61, no. 3, pp. 1179-1192, Mar 2014.

[7] Z. N. Low, R. A. Chinga, R. Tseng, and J. Lin, "Design and test of a high-power high-efficiency loosely coupled planar wireless power transfer system," IEEE Trans. Ind. Electron., vol. 56, no. 5, pp. 18011812, May 2009.

[8] C. R. Valenta and G. D. Durgin, "Harvesting wireless power: Survey of energy-harvester conversion efficiency in far-field, wireless power transfer systems," IEEE Microw. Mag., vol. 15, no. 4, pp. 108-120, Jun 2014.

[9] T. Sun, X. Xie, and Z. Wang, Wireless Power Transfer for Medical Microsystems, ser. SpringerLink : Bücher. Springer New York, 2013.

[10] N. A. Cruz and A. C. Matos, "The MARES AUV, a modular autonomous robot for environment sampling," in OCEANS 2008, Sep 2008, pp. 1-6.

[11] D. Oliveira, C. Duarte, V. G. Tavares, and P. G. de Oliveira, "Design of a current-mode class-D power amplifier in RF-CMOS," in Proceedings of the XXIV Conference on Design of Circuits and Integrated Systems (DCIS'2009), Zaragoza, Spain, Nov 2009, pp. 418-422.

[12] T. Nagashima, X. Wei, E. Bou, E. Alarcn, M. K. Kazimierczuk, and H. Sekiya, "Analysis and design of loosely inductive coupled wireless power transfer system based on class-E ${ }^{2}$ DC-DC converter for efficiency enhancement," IEEE Trans. Circuits Syst. I, vol. 62, no. 11, pp. 2781-2791, Nov 2015.

[13] S. Aldhaher, P. C. K. Luk, and J. F. Whidborne, "Tuning class E inverters applied in inductive links using saturable reactors," IEEE Trans. Power Electron., vol. 29, no. 6, pp. 2969-2978, Jun 2014.

[14] P. Amaral, C. Duarte, and P. Costa, "On the impact of timer resolution in the efficiency optimization of synchronous buck converters," International Journal of Power Electronics and Drive Systems, vol. 6, no. 4, pp. 693-702, Dec 2015.

[15] M. Kiani and M. Ghovanloo, "A figure-of-merit for designing highperformance inductive power transmission links," IEEE Trans. Ind. Electron., vol. 60, no. 11, pp. 5292-5305, Nov 2013.

[16] X. Zhang, L. E. Larson, P. M. Asbeck, and R. A. Langridge, "Analysis of power recycling techniques for RF and microwave outphasing power amplifiers," IEEE Trans. Circuits Syst. II, vol. 49, no. 5, pp. 312-320, May 2002.

[17] J. Love, RF Front-End: World Class Designs. Newnes, 2009. 